\title{
Myofascial Orofacial Pain Exacerbated after Masseteric Nerve Neurectomy
}

\author{
Hyun Nam¹, Daeun Ko ${ }^{1}$, Jin-Kyu Kang ${ }^{1,2}$, YoungJoo Shim ${ }^{1,2}$ \\ 'Department of Oral Medicine, Wonkwang University Daejeon Dental Hospital, Daejeon, Korea \\ ${ }^{2}$ Department of Oral Medicine, Wonkwang Dental Research Institute, School of Dentistry, Wonkwang University, Iksan, Korea
}

Received October 16, 2020

Revised November 6, 2020

Accepted November 6, 2020

Correspondence to:

YoungJoo Shim

Department of Oral Medicine, School of

Dentistry, Wonkwang University Daejeon

Dental Hospital, 77 Dunsan-ro, Seo-gu,

Daejeon 35233, Korea

Tel: +82-42-366-1128

Fax: +82-42-366-1115

E-mail: gc21@wku.ac.kr

https://orcid.org/0000-0001-7514-5974

This paper was supported by Wonkwang University in 2019.
Myofascial pain (MFP) is one of the most common causes of persistent orofacial pain. Patients with chronic myofascial orofacial pain may present with diffuse heterotopic pain, complicating the correct diagnosis. Treatment of chronic MFP should focus on the elimination of aetiologic factors. This article describes two cases of chronic MFP of the masticatory muscles, whose symptoms were exacerbated after masseteric nerve neurectomy. The patients had suffered from irrelevant treatment which did not resolve the symptom. Their symptom was managed by conventional treatment protocol. These cases emphasize the importance of correct diagnosis and evidence-based approach.

Key Words: Denervation; Masseter muscle; Myofascial pain syndrome

\section{INTRODUCTION}

Temporomandibular disorders (TMD) are the most common persistent orofacial pain [1], and myofascial pain (MFP) of masticatory muscles are the most common cause of all TMD [2]. Common symptoms of MFP in the jaw muscles include diffuse facial pain, restricted jaw function, tenderness, stiffness, and fatigue in the jaw muscles, referral of pain to the remote structures, and various sensory, motor and autonomic symptoms [2-4].

When correctly diagnosed, MFP generally responds well to conservative therapies, including physical self-regulation, exercise, physiotherapy, pharmacotherapy, and occlusal appliances [2,5]. However, referred pain caused by MFP often leads clinicians to make an incorrect diagnosis and perform unnecessary treatment [4]. We witnessed several patients with persistent orofacial pain who underwent masseteric nerve neurectomy to treat their symptoms and were thereafter exacerbated. Here, we present the diagnosis and treatment of those cases with a review of the related literature.

\section{CASE REPORT}

\section{Case 1}

A-48-year-old female presented with continuous pain on maxillary anterior teeth and facial pain. She had been suffering from the symptoms for 3 months. Two months before visiting the hospital, she underwent masseteric neurectomy to alleviate the pain. The procedure did not solve her symptoms and caused trismus.

She reported her pain 4 on a numeric rating scale (NRS, 0: no pain, 10: worst pain possible), and her pain site was both maxilliary anterior teeth and masseteric area. The pain was modified by jaw function. Dental examination and

Copyright (c) 2020 Korean Academy of Orofacial Pain and Oral Medicine. All rights reserved.

(c) This is an open-access article distributed under the terms of the Creative Commons Attribution Non-Commercial License (http://creativecommons.org/licenses/by-nc/4.0/), which permits unrestricted non-commercial use, distribution, and reproduction in any medium, provided the original work is properly cited. 
radiographic examination showed no dental or periodontal pathology on the anterior dentition. Muscle and TMJ examination showed tenderness on her both masseter, temporalis, and trapezius muscles. She reported of familiar facial pain with palpation of both masseter and temporalis muscles. Referral pain to the maxilliary anterior teeth was not duplicated on palpating both temporalis muscles. Her maximum unassisted opening (MUO) was restricted to $31 \mathrm{~mm}$, and maximum assisted opening (MA0) was restricted to 36 $\mathrm{mm}$. On occlusal examination, both central incisors and left premolar and molars were occluding in maximum intercuspal position (MICP). Her right premolars and molars were not in contact.

She was diagnosed as MFP with referral (according to diagnostic criteria for temporomandibular disorders [DC/TMD criteria]). She was instructed to perform Rocabado's $6 \times 6$ exercise [6] and received physical therapy every week. After 2 months of treatment, her pain and discomfort had been controlled and visited no more. Her MUO was increased to $41 \mathrm{~mm}$, and her right dentition (\#16,15,14 and \#46,45,44) was able to occlude in MICP.

\section{Case 2}

A-52-year-old female presented with continuous pain throughout right mandibular incisor to secondary molar region, trismus and malocclusion. She had been suffering from diffuse continuous aching pain for 21 months, and the pain was getting worse. Previous to the visit, she was examined by endodontists, periodontists and otolaryngologists and found no abnormality in her teeth, periodontium, neck and vocal cords. Four months before visiting the hospital, she was allegedly diagnosed as 'trigeminal neuralgia' and undergone masseteric nerve neurectomy at a dental clinic. This procedure worsened the pain and caused trismus and malocclusion (anterior open bite). She scored her pain 9 on NRS, and her pain was modified by mastication, speech and swallowing.

Dental examination and radiographic examination, including cone-beam computed tomography revealed no source of dental or periodontal pain and bone pathology. Muscle and TMJ examination showed severe tenderness to palpation on both TMJ, masseter, medial pterygoid and anterior digastric muscles. Referral pain to the right maxillary posterior region was duplicated on palpating right masseter muscle, and was familiar to her chief complaint. Her MUO and MAO was restricted to $36 \mathrm{~mm}$ and $40 \mathrm{~mm}$, respectively. Occlusal examination showed only her left and right second molars (\#17, 27, 37, 47) were occluding in MICP.

She was diagnosed as MFP with referral and TMJ arthralgia (according to DC/TMD criteria). Maxillary occlusal stabilization appliance was fabricated, and she was prescribed with non-steroidal anti-inflammatory drug (NSAID, aceclofenac $400 \mathrm{mg} \# 2 /$ day), muscle relaxant (tizanidine 3-6 mg\#3/day) and tricyclic antidepressant (TCA, nortriptyline

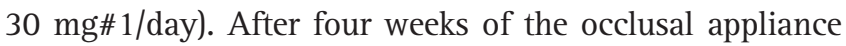
and pharmacologic therapy, her pain score was decreased to 4 on NRS. NSAID was discontinued from the fifth week; only muscle relaxant and TCA were given instead. Her pain on the right dentition and pain during speech and swallowing was almost completely diminished from the sixth week, reporting only a few instances of pain episode, with pain intensity decreased to 2 on NRS. Her MUO was increased to $40 \mathrm{~mm}$; however, the open bite was not solved.

Diagnostic cast was made to evaluate her dentition extraorally. Her MICP contact was sound in the study cast so selective grinding, or prosthetic treatment was postponed. Isometric exercises were taught to the patient in an attempt to strengthen the synergistic muscles of masseter [5]. Since 16th week (10th week after exercise) she did not report any pain, MUO was increased to $45 \mathrm{~mm}$, but her bite remained still. Selective grinding on \#16,17, 26, 27, 36, 37, 46, 47 was performed based on study model. However, open bite was relapsed after five days, and no further occlusal treatment had been made. She was instructed to perform isometric exercise and wear occlusal splint at night and given tizanidine $3 \mathrm{mg} \# 3 \mathrm{prn}$. She had been followed up for 32 months. Her pain and symptoms, including severe pain, mouth opening limitation and malocclusion had been controlled and discontinued the wear of splint. In her final visit, her left and right second premolars and first and second molars (\#15, 16, 17, 25, 26, 27, 35, 36, 37, 45, 46, 47) were occluding in MICP. 


\section{DISCUSSION}

MFP is a type of myogenous pain characterized by presence of the firm, hypersensitive bands or 'trigger points' in the muscle tissue [5]. Trigger points generate constant deep pain and may produce central excitatory effects and satellite trigger points [3,5]. Failure to resolve the trigger point often results in complex chronic pain condition in the orofacial region. Various conservative modalities such as physical self-regulation, exercise, physiotherapy, pharmacotherapy, and occlusal appliances are useful in the treatment of MFP [2,5]. Chronic MFP is often intractable, and pharmacotherapy helps alleviate the patient's discomfort $[2,5,7]$. NSAIDs are effective for acute pain, and muscle relaxants may convert an active trigger point into the latent state $[2,5]$. Antidepressants, especially TCAs, are one of the most widely used pharmacotherapy options.

Surgical intervention to the masseter muscle includes surgical excision and masseteric nerve neurectomy [8,9]. First introduced by Gurney in 1947 [10], surgical reduction of the masseter has been advocated by several authors to treat masseteric muscle hypertrophy [11-14]. Park et al. [12] described the application of radiofrequency coagulation in cosmetic masseter reduction and reported several complications, such as mucoserous fluid collections (4.5\%), late bleeding (3.0\%), infections (1.5\%), long-lasting pain (0.9\%), facial nerve injuries (0.6\%), Stensen's duct injuries(0.3\%) and limitation of temporomandibular joint movement $(1.8 \%)$. There were no reports on discomfort in mastication, decrease in masticatory force was observed in most of their subjects [12]. Surgical neurectomy of the masseteric nerve to treat masseter hypertrophy was first described in 2004 [15], and the application of radiofrequency rhizotomy was first reported in 2008 [9]. Both techniques were focused on the cosmetic issue and lacked long-term follow-up results, let alone the effects on the masticatory system.

Also, it should be noted that subjects of such reports are otherwise non-symptomatic, benign hypertrophy patients and their goal was a cosmetic improvement. Although the exact pathophysiology of chronic MFP in the orofacial region is unknown to date [16], current evidence suggests that it is related to the abnormalities in the pain modulation and central sensitization $[1,4,17]$. There is no evidence that masticatory muscle hyperactivities per se lead to the MFP of masticatory muscles [1]. Therefore, scientific and clinical evidence does not support masseteric nerve neurectomy or masseter reduction as a treatment option for the chronic masticatory MFP.

We have witnessed three more patients who underwent masseteric nerve neurectomy besides the presented cases (Table 1). Except for the patients \#3, all other patients underwent masseteric nerve neurectomy due to the orofacial pain. However, the procedure aggravated or generated orofacial pain and caused other complications, including mouth opening limitation and open bite. All the patients had persistent orofacial pain even after 3 to 24 months after the procedure. Especially, the patients \#3 has been having severe mouth opening limitation even after 10 months after the procedure.

Patients reported exacerbation of their symptoms after masseteric nerve neurectomy and complained heterotopic referred pain. Masseter muscle is essential for the fine jaw control and is required to bring the dentition into MICP [3]. One may argue that synergistic muscles might adapt to the functional need and take over the function of masseter. However, chronic MFP patients have low adaptive capacity

Table 1. Clinical manifestation after masseteric nerve neurectomy at the first visit

\begin{tabular}{|c|c|c|c|c|c|c|c|c|}
\hline Patient & Age $(y)$ & Sex & $\mathrm{CC}^{*}$ & CC at the first visit & CC duration** & $\mathrm{MUO}(\mathrm{mm})$ & $\mathrm{MAO}(\mathrm{mm})$ & $\begin{array}{l}\text { Occlusion at the } \\
\text { first visit }\end{array}$ \\
\hline 1 & 48 & Female & Orofacial pain & Orofacial pain & $3 \mathrm{mo}$ & 31 & 36 & Right posterior open bite \\
\hline 2 & 52 & Female & Orofacial pain & Orofacial pain, Trismus & $4 \mathrm{mo}$ & 36 & 40 & Anterior open bite \\
\hline 3 & 24 & Female & Aesthetic need & Orofacial pain, Trismus & $10 \mathrm{mo}$ & 14 & 16 & Left anterior open bite \\
\hline 4 & 24 & Male & Clenching & Trismus, open bite & $6 \mathrm{mo}$ & 32 & 33 & Anterior open bite \\
\hline 5 & 32 & Male & Headache & Headache, cervical pain & $24 \mathrm{mo}$ & 55 & Not performed & Anterior open bite \\
\hline
\end{tabular}

CC, chief complint; MUO, maximum unassisted opening; MAO, maximum assisted opening.

*Chief complaint at that time of undergoing masseteric nerve neurectomy; **Time elapsed since masseteric nerve neurectomy. 
in their masticatory and somatosensory system, and the pain modulation system is dysfunctional $[1,17]$. In those patients, if a muscle is compromised due to MFP, its synergist muscles which cooperate with involved muscle are overloaded and likely to develop MFP [18]. It was observed in Case 2, who had multiple MFP involvement on both sides of the masseter, medial pterygoid, and anterior digastric muscles. In such cases, the effect of irreversible neurectomy may be fatal.

Treatment of these patients should begin with the control of pain. Standard conservative therapies, including physical therapy, pharmacotherapy, and occlusal appliances were efficacious. In our experience, nortriptyline or amitriptyline $10-30 \mathrm{mg}$ at bedtime was the most effective for controlling complicated referred pain, like in the Case 2. Once the pain is reduced to a tolerable level, patients were instructed to perform gentle stretching of jaw muscles and isometric (or resistance) exercises of jaw muscles [5,6]. Isometric exercises not only relaxes the jaw muscles but also strengthens the muscles [5]. Therefore, it could facilitate the adaptation of masticatory muscles and alleviate the open bite. Irreversible treatment such as selective grinding seems to ineffective for the treatment of symptoms caused by masseteric neurectomy. In Case 2, open bite relapsed 5 days after the selective grinding. Once the pain is relieved, and the adaptation of masticatory muscles are completed, the patient's open bite was corrected without any occlusal treatment.

The critical difference between the Case 1 and Case 2 is the onset and duration of the pain. The longer the patient suffer from the pain, the more time is needed for the symptom resolution. Chronic pain causes central sensitization and loss of descending inhibitory system [17], contributing the alteration and dysfunction of the masticatory system. These neuroplastic changes cause the patient less responsive to the treatments [1]. However, this does not imply that the patient is incurable; conservative treatments can manage their symptoms.

In this article, we discussed the exacerbation of orofacial pain after masseteric nerve neurectomy. Finding the source of chronic orofacial pain is often challenging due to its complexity. Thorough history taking and differential diagnosis are required to avoid unnecessary treatment. Even in those complicated cases, conservative and reversible therapies were able to achieve the resolution of symptoms. Evidence-based conservative treatment should always be used.

\section{CONFLICT OF INTEREST}

No potential conflict of interest relevant to this article was reported.

\section{ORCID}

\author{
Hyun Nam \\ https://orcid.org/0000-0002-5929-4109 \\ Daeun Ko \\ https://orcid.org/0000-0003-1300-8143 \\ Jin-Kyu Kang \\ https://orcid.org/0000-0001-7668-7468 \\ YoungJoo Shim \\ https://orcid.org/0000-0001-7514-5974
}

\section{REFERENCES}

1. Benoliel R, Svensson P, Heir GM, et al. Persistent orofacial muscle pain. Oral Dis 2011;17 Suppl 1:23-41.

2. Scrivani SJ, Keith DA, Kaban LB. Temporomandibular disorders. N Engl J Med 2008;359:2693-2705.

3. Simons DG, Travell JG, Simons LS. Travell \& Simons' myofascial pain and dysfunction. Vol. 1. Upper half of body. Baltimore: Williams \& Wilkins; 1999. pp. 1038.

4. Masuda M, Iida T, Exposto FG, et al. Referred pain and sensations evoked by standardized palpation of the masseter muscle in healthy participants. J Oral Facial Pain Headache 2018;32:159166.

5. Okeson JP. Management of temporomandibular disorders and occlusion. 7th ed. St. Louis: Elsevier/Mosby; 2013.

6. Rocabado M, Iglarsh Z. Physical modalities and manual techniques used in the treatment of maxillofacial pain. In: Rocabado M, Iglarsh Z, eds. Musculoskeletal approach to maxillofacial pain. Philadelphia: Lippincott; 1991. pp. 187-192.

7. Haviv Y, Zini A, Sharav Y, Almoznino G, Benoliel R. Nortriptyline compared to amitriptyline for the treatment of persistent masticatory myofascial pain. J Oral Facial Pain Headache 2019;33:7-13.

8. Huang JL, Chen G, Chen XD, Zhou BR, Luo D. A comparative study of the efficacy and safety of radiofrequency ablation and botulinum toxin A in treating masseteric hypertrophy. Exp Ther Med 2014;7:1203-1208.

9. Kwon J, Lee M, Lee K, Choi M. Masseter muscle reduction using radiofrequency rhizotomy. J Korean Soc Aesthet Plast Surg 2008;14:43-48. 
10. Gurney CE. Chronic bilateral benign hypertrophy of the masseter muscles. Am J Surg 1947;73:137-139.

11. Beckers HL. Masseteric muscle hypertrophy and its intraoral surgical correction. J Maxillofac Surg 1977;5:28-35.

12. Park Y, Jo Y, Bang S, et al. Radiofrequency volumetric reduction for masseteric hypertrophy. Aesthetic Plast Surg 2007;31:42-52.

13. Rispoli DZ, Camargo PM, Pires JL Jr, Fonseca VR, Mandelli KK, Pereira MAC. Benign masseter muscle hypertrophy. Braz J Otorhinolaryngol 2008;74:790-793.

14. Ham JW. Masseter muscle reduction procedure with radiofrequency coagulation. J Oral Maxillofac Surg 2009;67:457-463.

15. Hwang K, Kim YJ, Park H, Chung IH. Selective neurectomy of the masseteric nerve in masseter hypertrophy. J Craniofac Surg 2004;15:780-784.

16. International Classification of Orofacial Pain, 1st edition (ICOP). Cephalalgia 2020;40:129-221.

17. Botelho LM, Morales-Quezada L, Rozisky JR, et al. A framework for understanding the relationship between descending pain modulation, motor corticospinal, and neuroplasticity regulation systems in chronic myofascial pain. Front Hum Neurosci 2016;10:308.

18. Gerwin RD. Classification, epidemiology, and natural history of myofascial pain syndrome. Curr Pain Headache Rep 2001;5:412420. 